\title{
A multiple case study of the personal values of counselor trainees from a psychological flexibility perspective: a sample from Turkey
}

\author{
Yasin Aydın ${ }^{1}$ (D) Merve Usta ${ }^{2}$ \\ Accepted: 3 December 2020 \\ (C) The Author(s), under exclusive licence to Springer Science+Business Media, LLC part of Springer Nature 2021
}

\begin{abstract}
Many different approaches have been used in psychology to determine values and investigate valuing behavior. However, understanding the individual significance of the values is crucial to predict the actions that produce them. A significant number of studies exist in counseling education that focuses on the diverse issues of counselor trainees. These include, but are not limited to, self-efficacy levels, emotional awareness, attachment styles, and supervision experiences in counseling education. During the literature review conducted for this study, it was discovered that there was no investigation of counselor trainees' values from an Acceptance and Commitment Therapy (ACT) perspective. Using ACT as the overarching framework, the current qualitative study aimed to examine counselor trainees' values from a psychological flexibility perspective. During the data collection process, all counselor trainees $(N=62)$ were initially asked to fill both an Acceptance and Action Questionnaire (AAQ-II) that measured their psychological flexibility level and a Valuing Questionnaire (VQ) that measured their valuing levels. Participants were then split into two groups, based on their AAQ and VQ scores regarding either high or low psychological flexibility or values awareness. As a result of the analysis, 18 ( 15 female, three male) senior counseling students took part in the study, with the data being collected via focus groups and individual interviews. As a result of the study, four main themes were identified following detailed content analysis: (1) perception of values, (2) committed action vs. experiential avoidance moves, (3) present movement awareness and perspective skills, and (4) cognitive defusion and fusion experience. Implications were discussed in light of the relevant literature.
\end{abstract}

Keywords Psychological flexibility $\cdot$ Personal values $\cdot$ Counselor trainees $\cdot$ Case study

\section{Introduction}

Human behavior is shaped by purpose and personal values, and those values are unique to each human being (Dahl et al., 2009). Personal values can also be considered one of the most powerful motivators in life. Individuals are likely to experience many difficulties when their life does not have meaning or purpose (Garfield, 1992). The phenomenon of personal

Yasin Aydın

yasnaydin@gmail.com

Merve Usta

merve.usta@outlook.com.tr

1 Department of Educational Sciences, Counseling and Guidance Department, Faculty of Education, Bolu Abant İzzet Baysal University, Golkoy Campus, Bolu, Turkey

2 Department of Educational Sciences, Counseling and Guidance Department, Faculty of Education, Hasan Kalyoncu University, Gaziantep, Turkey values has been studied via various approaches, ranging from humanistic psychology to a self-actualization process (Rogers, 1964), positive psychology (Sheldon et al., 2002), and the behavioral approaches of Skinner (1971), which are defined in terms of contingencies and reinforcements. Personal values have also been described extensively by Schwarts (2005), with a focus on the social psychology perspective, and by Rokeach (1973) with an emphasis on instrumental and terminal values definitions. It is clear from the above that personal values have been conceptualized from different perspectives. Nevertheless, there is a theoretically derived behavioral approach, named Acceptance and Commitment Therapy (ACT) (Hayes et al., 1999). There is promising empirical support that this modern approach accurately describes values or valuing behavior.

Acceptance and Commitment Therapy (ACT), which is considered one of the third wave cognitive-behavioral approaches, explain human behavior from a consideration of acceptance and mindfulness perspectives within the core concept of psychological flexibility (Hayes et al., 1999). 
Psychological flexibility is defined as experiencing emotions, thoughts, or past events without trying to change them. It allows one to be conscious of the present moment based on the value of interventions in acceptance, commitment, and behavior (Hayes \& Lillis, 2012). The psychological flexibility model is focused on six consistently interrelated core processes. Therefore, psychological flexibility can be seen as a unified model with six-core dynamic features that explain both human adaptability, psychopathology, and suffering (Hayes et al., 2012). The interrelated and dynamic six-core processes, which can be conceptualized on a hexagon, are (1) Flexible attention to the present moment, (2) Clarified values, (3) Actions or behavior according to chosen values, (4) Awareness of oneself that goes beyond content, (5) Being able to defuse from thoughts or other private events, (6) Opening up and willingness (Hayes et al., 2012).

Psychological flexibility in ACT emphasizes the importance of moving towards personal values that are defined as being chosen directions in life (Hayes \& Smith, 2005). Valuing, on the other hand, refers to the actions taken to implement freely chosen purposes in life that are important to one's sense of self (Hayes et al., 1999). Therefore, it is essential that individuals are encouraged to choose what is important to them or what they want their lives to be about (Wilson \& Murrell, 2004). According to ACT, values are different from life goals, which are desired attainable end-states. Values, on the other hand, guide individuals towards attaining meaningful existence (Hayes et al., 1999).

Values assessment, in accordance with other psychological flexibility processes, is one of the core issues in ACT. Clarifying the "values- behavior" discrepancy is often considered an initiating force in the behavior change process (Hayes et al., 2012). To gain a better understanding of this discrepancy or gap between values and actions, Wilson, Sandoz, Kitchens, and Roberts (2010) developed the Valued Living Questionnaire (VLQ). This questionnaire involves ten-value areas: family relations, marriage/couples, intimate relations, parenting, friendships/social interactions, employment, education/training, recreation, spirituality, citizenship/community life, and physical well-being. VLQ primarily assesses the importance of personal values for the individual on a scale from 1 (not at all important) to 10 (very important). It then asks participants to score the consistency of their importance scores, based on the actions taken by each value area. This process allows individuals to easily determine the discrepancy or gap for each value area (Wilson et al., 2010). Values can also be considered evolving patterns of dynamic actions that most probably vary over time (Dahl et al., 2009). In this regard, Smout, Davies, Burnst, and Christie (2014) developed the Valuing Questionnaire (VQ) and emphasized that valued living can be understood and assessed, not only by focusing on various life domains but also by the extent to which individuals live out what they value in day-to-day life.
It is vital to appreciate the relationship between actions based on personal values, as well as those based on psychological distress, because it may be difficult for individuals to act in accordance with freely chosen values when there is the presence of a psychological condition such as anxiety, depression, or situational stress (Hernandez, 2013). This is because individuals may move away from what they value personally by attempting to avoid having negative feelings, thoughts, and sensations resulting from psychological disturbance. This phenomenon is called experiential avoidance (EA) (Hayes et al., 1996) and is simply defined as the attempts to avoid internal private experiences, including thoughts, feelings, memories, and physical sensations. However, such avoidance can lead to harm and damaging conclusions in the long run (Hayes \& Smith, 2005). In other words, EA arises when someone has difficulty adhering to their values. For example, suppose a person with social anxiety avoids social interactions and public gatherings because of the negative or undesired feelings, thoughts, and sensations they evoke. In that case, this person may move away from his/her valued direction in life. This avoidance may relieve in this person in the short term, but it is a clear example of an EA move and will definitely prevent $\mathrm{him} / \mathrm{her}$ away from adhering to values in the mid and long run.

There is a growing body of evidence showing that experiential avoidance (EA) is strongly linked to psychopathology and behavioral problems (Kashdan \& Rottenberg, 2010), psychological distress and reduced quality of life (Hayes et al., 2004), anxiety and depression (Forsyth et al., 2003; Kashdan et al., 2009; Tull et al., 2004) and chronic pain (Costa \& PintoGouveia, 2013; Zettle et al., 2005). EA was also studied within the college population, including a focus on childhood psychological abuse (Reddy et al., 2006), alcohol-related problems (Levin et al., 2012), and college adjustment (Aydın, 2016).

\section{Values of Counselor Trainees (CT)}

The counseling profession is a helping relationship to empower individuals, families, and groups to accomplish their mental, educational, and career goals (American Counseling Association [ACA], 2010). It requires counselors to undergo many stages, including extensive training and supervised practice (Gladding, 2017). This long process can be very exhausting for the counselor trainees (Skovholt, 2012). Furthermore, according to Skovholt and McCarthy (1988), novice counselors may experience a number of critical incidents that can permanently influence their understanding of the counseling profession.

Counselor education in Turkey is mainly only studied at the undergraduate level (Korkut, 2006). Therefore, students graduate with a bachelor's degree from counseling programs at approximately the age of 22 and are expected to work as counselors, mostly within an educational setting. In recent 
years, counselor trainees in Turkey have encountered various difficulties, including, but not limited to, recognition of the counseling profession by the authorities and public, increased unemployment rate, and a lack of further educational opportunities. These problems can lead to undesirable thoughts and feelings for trainee counselors that may be difficult to overcome.

With this theoretical framework in mind, and because counselor trainees experience various difficulties in relation to their profession and current developmental and personal issues, the purpose of this study is to explore the personal values of counselor trainees from the psychological flexibility perspective. For this purpose, 18 counselor trainees were selected based on their psychological flexibility and personal values clarification levels. The following research questions were used in the investigation:

1. How do counselor trainees describe their personal values from a psychological flexibility perspective?

2. How do counselor trainees act according to their chosen values?

\section{Method}

A multiple case study was implemented (Creswell, 2013) to investigate counselor trainees' personal values from a psychological flexibility perspective. According to Yin (2003), one should basically conduct a case study to answer "how" and "why" questions, as well as to understand the contextual conditions of a phenomenon related to cases and to appreciate when the boundaries between phenomenon and context are unclear. In this current research, the case study aimed to understand counselor trainees' true value experiences from a psychological flexibility perspective with high and low levels of clarified values.

\section{Sampling}

The participants ( 15 females and three males with ages ranging from 21 to 24) were senior students in the Department of Counseling and Guidance, a four-year undergraduate program at a state university in western Turkey. The four-year counseling and guidance program offers students intensive courses on various counseling topics while also providing practical experience in the senior year. Data was collected through convenience sampling, and participants were recruited among all senior counseling students $(N=62)$ via evaluating the results of psychological flexibility (AAQ-II) and valuing questionnaire (VQ). In terms of determining the number of participants to include in a case study, Creswell (2013) states that at least four or five cases should be included, even if there is no clear answer to the question. In terms of the gender balance of the participants, although the majority of the CTs among all senior counseling students were female ( 50 females, 12 males), the sample did seem to represent all CTs. The data was collected by researchers between January and April 2019.

\section{Data Collection Procedure}

Permission was obtained from the Human Research Ethics Committee before any data was collected. The data collection process in this study was threefold. Firstly, all senior counselor trainees $(N=62)$ at the university were asked to complete the Acceptance and Action Questionnaire (AAQ-II) in order to measure psychological inflexibility levels, and the Valuing Questionnaire (VQ) to measure current values. The aim was to identify participants with high and low psychological inflexibility levels and personal values to divide them into two different homogenous focus groups.

Acceptance and Action Questionnaire (AAQ-II) AAQ-II is a 7item scale that was developed by Bond et al. (2011) to measure psychological inflexibility. The Cronbach alpha of the scale was 0.88 , with a one-factor solution. AAQ-II was translated into Turkish by Yavuz et al. (2016), and the Turkish version of the scale also provided a one-factor solution with 0.84 Cronbach alpha.

Valuing Questionnaire (VQ) VQ was developed by Smout et al. (2014) to measure individuals' current valuing status. VQ is a 10-item scale with a two-factor structure of progress and obstruction. The Turkish adaptation of the VQ was conducted by Aydın and Aydın (2017), and a two-factor structure of the scale was confirmed using Cronbach alpha 78 .

As a subsequent second step, all counselor trainees $(N=$ 62) were asked to fill values clarification forms (VCF) based on the ten value areas determined by Wilson et al. (2010). During this exercise, the trainees were asked to think and write about the most important personal values that existed in their lives. They also were specifically asked to score the importance (I) of each value and the consistent, committed actions (C) that they took according to those personal values. Finally, candidates were also expected to specifically declare each of their values on the values clarification forms. As a result of completing the values clarification forms (VCF), the importance of each participant's personal value (I) and consistent, committed actions (C) in relation to the personal values are presented in Table 1. VCFs were discussed in detail in focus groups and individual interviews as the last step of data collection. The third step of the data collection was mainly semistructured interviews via two focus groups (one group with the participants of a high level of psychological flexibility and clarified values) and the other group with a low level of psychological flexibility unclarified values). As a part of the third 
Table 1 Focus Group with High and Low Psychological Flexibility and Values Awareness

\begin{tabular}{|c|c|c|c|c|c|c|c|c|c|c|c|c|c|c|c|c|c|c|c|}
\hline Participant Value Area & I vs $\mathrm{C}$ & FH1 & $\mathrm{FH} 2$ & FH3 & FH4 & FH5 & MH1 & MH2 & MH3 & FHi1 & FHi2 & FL1 & FL2 & FL3 & FL4 & FL5 & FL6 & FLi1 & FLi2 \\
\hline \multirow[t]{2}{*}{ Family Relations } & I & 7 & 10 & 9 & 10 & 8 & 10 & 9 & 10 & 10 & 10 & 9 & 10 & 9 & 10 & 10 & 10 & 10 & 8 \\
\hline & $\mathrm{C}$ & 7 & 8 & 8 & 8 & 9 & 7 & 7 & 9 & 10 & 8 & 7 & 5 & 9 & 8 & 8 & 8 & 6 & 5 \\
\hline \multirow{2}{*}{$\begin{array}{l}\text { Intimate Relation- } \\
\text { ships }\end{array}$} & I & 8 & 10 & - & 10 & 3 & 10 & 8 & 9 & 9 & 10 & - & - & - & - & - & - & - & - \\
\hline & $\mathrm{C}$ & 5 & 0 & - & 8 & 2 & 6 & 3 & 4 & 10 & 10 & - & - & - & - & - & - & - & - \\
\hline \multirow{2}{*}{$\begin{array}{l}\text { Friends/ } \\
\text { Social Life }\end{array}$} & I & 8 & 8 & - & 9 & 9 & 10 & 9 & 9 & 9 & 10 & 9 & 8 & 8 & 9 & 7 & 8 & 10 & 9 \\
\hline & $\mathrm{C}$ & 7 & 7 & - & 8 & 9 & 7 & 9 & 8 & 8 & 9 & 7 & 2 & 7 & 8 & 6 & 6 & 9 & 7 \\
\hline \multirow[t]{2}{*}{ Employment } & I & 8 & 7 & 5 & 10 & 7 & 8 & 8 & 10 & 9 & 10 & 9 & 8 & 6 & 8 & 8 & 10 & 10 & 8 \\
\hline & $\mathrm{C}$ & 8 & 5 & 5 & 5 & 5 & - & 7 & 7 & 8 & 9 & 4 & 2 & 8 & 8 & 5 & 8 & 5 & 5 \\
\hline \multirow[t]{2}{*}{ Education/ Training } & I & 7 & 10 & - & 10 & 10 & 8 & 10 & 9 & 9 & 10 & 9 & 8 & 10 & 10 & 7 & 10 & 10 & 9 \\
\hline & $\mathrm{C}$ & 7 & 6 & - & 5 & 5 & 5 & 8 & 8 & 9 & 9 & 7 & 5 & 8 & 7 & 6 & 7 & 5 & 6 \\
\hline \multirow[t]{2}{*}{ Recreation } & I & 8 & 10 & 9 & 7 & 7 & 9 & 9 & 7 & 9 & 9 & 8 & 8 & 7 & 7 & 7 & 6 & 10 & 7 \\
\hline & $\mathrm{C}$ & 6 & 10 & 8 & 5 & 9 & 8 & 6 & 2 & 10 & 9 & 8 & 1 & 8 & 2 & 7 & 8 & 4 & 7 \\
\hline \multirow[t]{2}{*}{ Spirituality } & I & 8 & 10 & - & 10 & 3 & 8 & 9 & - & 9 & 10 & 2 & 8 & 10 & 10 & 10 & 8 & 10 & 10 \\
\hline & $\mathrm{C}$ & 6 & 7 & - & 6 & 0 & 6 & 7 & - & 9 & 8 & 0 & 5 & 8 & 6 & 7 & 6 & 6 & 8 \\
\hline \multirow[t]{2}{*}{ Physical Well-Being } & I & 8 & 10 & - & 10 & 2 & 6 & 10 & 10 & 10 & 9 & 10 & 10 & 8 & 10 & 8 & 8 & 10 & 8 \\
\hline & $\mathrm{C}$ & 6 & 7 & - & 5 & 2 & 6 & 8 & 5 & 8 & 8 & 4 & 9 & 5 & 5 & 6 & 8 & 6 & 5 \\
\hline
\end{tabular}

( $\mathrm{F}=$ Female, $\mathrm{M}=$ Male, $\mathrm{H}=$ High, $\mathrm{L}=$ Low, $\mathrm{i}=$ Individual)

step, two participants from each group (high/low) were randomly selected and interviewed individually to maintain the accuracy of the data.

Semi-structured interview questions were formed based on six core components and commonly used protocols in the ACT approach. The interview questions included the values process of 'What and who matters to you most?', the committed action process of 'What are the behaviors that help you to move towards your values?', the cognitive defusion process of 'What are the internal unwanted/undesired feelings, thoughts, and sensations that get in the way of your values?', the acceptance process of 'What do you do when difficult, undesired feelings, thoughts, and sensations occur?', the present moment awareness process of 'What do you notice in your mind/body when you pay attention of your experiences?', and the self-as-context process of 'What does it feel like to move/not to move in the direction of your values?'. While the focus group interviews took approximately $90 \mathrm{~min}$, the individual interviews took an average of $20 \mathrm{~min}$. The two researchers conducted the interviews at the university's counseling clinic, and all of them were recorded with audio devices.

\section{Data Analysis}

Before moving on to the focus groups and individual interviews stage, it was first crucial to better understand the level of both CT's values and psychological flexibility. Therefore, the responses obtained from AAQ-II and VQ questionnaires were the first step in understanding the values and psychological flexibility level of 18 CTs from a quantitative perspective.
The content analysis method was utilized in this study since the coding process was implemented on language units observed within the verbatim transcripts gathered from both focus groups and individual interviews (Krippendorff, 2004). The content analysis was systematically analyzed as deductive in nature, as the psychological flexibility approach provides clear guidelines. During the analysis, the participants with the highest and lowest psychological flexibility levels were split into two different homogeneous groups. The data was gathered from the focus groups and individual interview processes following the psychological flexibility core procedure. The content analysis procedure included several steps. First, the transcripts were carefully and independently read several times by the researchers. Categories and themes were selected based on the literature review presented above, and then the categories and themes were determined following consensus being achieved amongst the researchers.

Regarding validity and reliability, the researchers applied several methods, which included data triangulation, member checking, spending prolonged time in the field (Creswell, 2013), and intercoder reliability of Krippendorff's $\alpha$ (KALPHA) value (Table 2). The data triangulation was ensured by both collecting data from focus groups and individual interviews. Individual interviews, separate from the focus groups, were conducted to overcome possible respondent or social desirability bias in the focus groups. Member checking was another strategy used to check the accuracy of the findings. The member checking process entailed three participants from each group (high and low) being invited to check the study's categorical and thematic outcomes. As the researchers 
Table 2 Overall Categories and Themes and Intercoder reliability

\begin{tabular}{llll}
\hline Sample Questions & Themes & Categories & $\begin{array}{l}\text { Intercoder } \\
\text { reliability }\end{array}$ \\
\hline "How important is this personal value to you?" & Perception of values & $\begin{array}{l}\text {-Clear values } \\
\text {-Nonclarified goal directed value perception }\end{array}$ \\
$\begin{array}{l}\text { What do you do to move towards your important } \\
\text { values? "How functional are your steps?" }\end{array}$ & $\begin{array}{c}\text { Committed action vs } \\
\text { experiential avoidance } \\
\text { "What do you notice in your mind and body } \\
\text { when you try to move towards your personal values?" } \\
\text { "What kinds of undesired feelings and thoughts } \\
\text { occur when you try to move towards your personal values?" }\end{array}$ & $\begin{array}{c}\text {-Small steps in the service of values } \\
\text {-Expent moment awareness avoidance actions }\end{array}$ & $\begin{array}{l}\text {-Mindful awareness } \\
\text { fusion experience }\end{array}$ \\
\hline
\end{tabular}

had more than eight years of experience with the participants within the same setting, this meant that the findings were more accurate.

The research team consisted of three individuals: the first being an author and faculty member with training and experience in the qualitative studies and third-wave cognitive-behavioral approaches, including the ACT. The second member of the team is also an author and a counselor education doctoral student with qualitative study and training experience in the ACT. The research team's final member is a specialist counselor with more than ten years of experience in qualitative studies and counseling college students.

\section{Results}

This study was based on the qualitative research method and yielded a large amount of information about counselor trainees' experiences regarding their values and related actions. As a result of detailed analysis based on the research questions stated above, themes and categories were determined. The related findings are structured below systematically based on the themes and categories.

Qualitative findings were supported in this study by quantitative ones by providing consistency scores for each case, based on Values Clarification Forms (VCF). Before the focus group and individual interviews, each participant evaluated the importance of his/her personal values (I) on a scale of 0 10 ( 0 being not important at all and 10 being very important) and consistent, committed actions (C) on a scale of $0-10(0$ being not all consistent with my value, ten being completely consistent with my value). The findings (Table 1) revealed major differences between the cases who were in the high $(\mathrm{H})$ group (cases with a high level of psychological flexibility and clear personal values) and the low (L) group (cases with a low level of psychological flexibility and clear personal values). On the other hand, no major differences were indicated in terms of gender, age, or current academic success, based on the statements provided.
When the importance of personal values (I) and consistent, committed actions (C) scores in Table 1 are examined, it can be seen that the discrepancies between the I and C scores in the high group are less than those of the low group. In other words, the subjects with high psychological flexibility and clear personal values tend to act more in the service of those values. For instance, when we compare the 'employment' value scores for both the high and the low groups (Table 1), while the students in the high group mostly place sufficient importance on employment values and act accordingly (FH1: 8/8, FHil: $9 / 8$ ), those in the low group seem to mostly be the opposite (FL2: 8/2, FLi2: 8/5). The results of the I and C scores of the counselor trainees provide some important insights about their values and committed actions. However, it is vital to look at the content analysis results to better understand the $\mathrm{I}$ and $\mathrm{C}$ scores.

As a result of content analysis, four themes, and related categories for each theme emerged from this current study (Table 2). The themes were (1) Perception of values, (2) Committed action vs. experiential avoidance moves, (3) Present movement awareness skills, and (4) Cognitive defusion and fusion experiences. All of the data was transcribed and re-coded in the context of these emerged themes.

\section{Perception of Personal Values}

The participants' understanding and perception of their personal values emerged as one of the basic themes. After the recoding, the perception of the personal values theme was completed; two major categories: (a) Clear personal values and (b) Non-clarified goal-directed value perception became apparent. A female counselor trainee from the high group (FL2) voiced her understanding of family values as: 'I care about my family because they will always be with me till I die,' which was a good example of clear values. On the other hand, another female counselor trainee from the low (FL1) group stated: 'I always had high goals in my life. University was the biggest one. I put a lot of effort to achieve this goal. My next goal after graduation is to find a job. But I'm not sure how this 
will happen. This situation is frankly a cause of distresses'. This direct quote demonstrates a goal-oriented value perception, which, in turn, creates stress and pressure.

\section{Committed Action Vs. Experiential Avoidance Moves}

Counselor trainees expressed their thoughts and feelings concerning the actions they take or commitments they make in the focus group and individual interviews. Following an analysis of the content produced, it was seen that the actions of the participants were either in the service of their personal values or toward EA moves. Two major categories based on this theme emerged: (a) Small steps in the service of personal values and (b) Experiential avoidance actions. One male participant (MH2) stated that: 'I value my family a lot. Even though I live in another city, I call them and share my problems whenever I need to'. While the above statement of MH2 is an example of Category A (small steps in the service of values), the following statement from a female participant (FL6) is a good example of an experiential avoidance move. So, considering the meaning of experiential avoidance, it is an example of avoiding meaningful activities to prevent unwanted internal events. 'I care about my social life and friends. However, I often think that others are better than me and so don't go out'. Another type of experiential avoidance move was expressed by a female participant from a low group (FL3). 'I usually find myself spending hours on the smartphone and social media when I want to start studying. I am always procrastinating'.

\section{Present Moment Awareness Skills}

Being aware of one's current state of mind and physical sensations are one of the core issues in personal values. In terms of the theme, (a) mindful awareness, and (b) a lack of mindfulness, emerged as two categories. It was mainly statements of participants in the high group, which indicated some awareness of the present moment, both mentally and physically. Three participants (FH2, MH2, and FHi2) all agreed that they were aware of their current thoughts and feelings in daily life. For instance, a female participant interviewed individually (FHi2) stated that 'Social interactions are very important for me. When I feel bad, I usually notice that my body also reacts in the same way. I have experienced these kinds of feelings a lot recently because I feel under pressure about the future. But I think I am not the only one!'. On the other hand, a female participant from the low group (FL4) complained that 'I have bad headaches, especially when I think of the aftergraduation period. It is bad that I cannot control my headaches' which perfectly shows how goal-oriented understanding can cause a lack of awareness of the present moment.

\section{Cognitive Defusion and Fusion Experiences}

Being able to distance oneself from internal private feelings, thoughts, and sensations is one of the most important abilities one can have in terms of psychological flexibility. As participants stated their experiences based on values and related actions, most of them, both in the high and the low groups, provided multiple insights related to cognitive defusion and fusion strategies. Some of the participants learned from their internal private feelings, thoughts, and sensations (Category A), while others were less successful in doing that (Category B). One of the female participants' (FH1) statement was a good example of concluding internal feelings, thoughts, and sensations. The participant stated that 'I used to go to the gym for my physical well-being and felt that it was something great to do. However, I have stopped going, and now I notice that I feel guilty'. On the other hand, another participant (FL1) from a low values clarification group stated that 'I don't want to get into that vicious cycle in where new graduates become trapped in a very low paying position with no chance for self-growth. That really scares me'.

In addition to the themes and categories, the current study also revealed some very important findings in relation to the above results in terms of content analysis. If we first consider counselor trainees with high psychological flexibility, it is seen that they mostly have high-value awareness in comparison to others. This means that they seem to be more willing to create some room for undesired feelings, thoughts, and sensations. Secondly, it is seen that stagnation in one area of personal values appears to be compensated by progress in another value area. This finding is supported by the following statement from a male participant (MH1): 'The importance I attach to one value increases while others seem to decrease. I think this is very natural because I am spending my time on it now'. Thirdly, personal values are not disconnected areas of life but are rather an inseparable part of it. In other words, there is a 'domino effect' between values, and most of the counselor trainees in both high and low groups agreed that personal values are connected. The statement of a male participant (MH2) was a clear expression of this finding: 'You know dominos? When you just touch one of them, others start to fall, and then a big picture emerges. For me, that first domino is absolute "health" because if I am unhealthy, my career, job, and occupation don't matter.'

Finally, according to the findings, CT's sense of self matters in both high and low groups. CTs in the high group revealed mostly a positive sense of self by making statements such as the following 'I feel like I am on the same page with most of my friends in this group. I also believe in myself! I would like to become a good counselor, and I know it takes time. I might do some other stuff for some time. However, it does not mean that I will not work hard on becoming a good counselor (MH2)'. 


\section{Discussion}

The Acceptance and Commitment Therapy (ACT) and the psychological flexibility model emphasizes the vitality of actions directed by personal values in providing a better understanding of human behavior. In this regard, this study's main purpose was to examine how clear the counselor trainees (CT) are about their personal values from a psychological flexibility perspective and how well they can act in the service of those values. The findings are systematically discussed below in the light of two basic research questions, and four themes emerged as a result of content analysis.

Firstly, the results of this study revealed that CTs with high levels of psychological flexibility and clarified personal values tend to be more engaged in life in comparison to those CTs in the low group. The ACT model proposes that individuals who have clear values and meaning in life can take steps towards their personal values (Hayes et al., 2012). The findings in the themes of "perception of personal values" and "committed action vs. experiential avoidance moves" seemed to support the major proposition of ACT and existing knowledge. Regarding the clarified personal values and the corresponding actions taken, previous research revealed similar findings in terms of understanding the connection between values and committed actions. For example, undergraduate students with a low level of personal values, as measured by VLQ (Wilson et al., 2010), reported more psychological distress and experiential avoidance (Van Dyke, Rogers, \& Wilson, 2006). Similarly, Adcock et al. (2007) investigated the relationship between having clear personal values, clinical distress, and the mediating effect of experiential avoidance with 388 undergraduate students. They found that personal values were a strong and significant predictor of well-being in college students.

Secondly, CTs in the high group seemed to be more mindful than those in the low group. This finding is consistent with the previous research since being consciously aware and noticing current feelings, thoughts, sensations, and actions with a non-judgmental attitude is a supportive factor of freely chosen values. In this regard, Vilardaga et al. (2011) conducted research with 699 addiction counselors in the US to investigate the roles of mindfulness and values-based actions on their burnout level. The results indicated that mindfulness and values-based actions had a strong and consistent relationship with burnout. It can also be said that mindful awareness in the service of personal values provides more psychological flexibility and less burnout in counselors. It is, therefore, possible to conclude, to some degree, that CTs in the high group had more daily connection to their values than those in the low group. Thirdly, CTs in both the high and low groups seemed to have the problem of being overwhelmed by their internal private feelings, thoughts, and sensations while trying to act upon their personal values. Cognitive fusion, which can be defined as dealing with such personal emotions literally rather than just viewing them as being merely thoughts, feelings, and sensations, can be very detrimental. Kraft et al. (2018) performed with a college student sample showed that cognitive fusion was a predictor of distress, depression, generalized anxiety, hostility, academic distress, and student role problems. On the other hand, cognitive defusion was also strongly related to an effective coping strategy for stress in a study conducted with adolescents (Donald et al., 2016).

The study's additional findings in this paper revealed that CTs in the high group mostly seemed to have greater psychological flexibility than those of the low group. One of the most pervasive findings of the current study was that CTs in both groups emphasized the interactive nature of values. This major finding is consistent with the value-driven and dynamic nature of the ACT approach. Hence, the key aspect of Schwartz's (1992) values theory assumed a structural and relational pattern among values (Bardi \& Schwartz, 2003).

Given that personal values are life domains and a major driving force for behavior change in the ACT model, college counseling centers can provide workshops, seminars, and psychoeducation groups, not only for CTs but also for all college students, to provide a better understanding of personal values. It is now obvious that there is a general move toward online programs. In this regard, CTs can take advantage of ACT-based online professional programs before they embark upon their career. Levin et al. (2016) found that web-based ACT helped college students reduce their overall distress and anxiety while generally improving their mental health. Another implication that can be drawn from the current study is the difference between value and goal-oriented cultures. CTs mainly go through western (individualistic) oriented counseling training in Turkey. On the other hand, society in Turkey can be best described as a Eurasian (mostly collectivist) culture. The discrepancy between the nature of the training they receive and the culture participants live in can sometimes cause certain contradictions in personal values. Therefore, CTs can provide some extra courses to clarify and work on underlying values consistent with the local culture.

There are several limitations to the current study. Firstly, it was conducted with only 18 CTs from one university. Therefore, the findings cannot be generalized to all CTs. Further studies that involve more participants from different universities that may eventually lead to different themes and categories should, therefore, be taken into consideration. Secondly, this study explored the importance of values from a psychological flexibility perspective, emphasizing the personal valuing processes. However, values cannot be sufficiently understood without investigating the other five psychological flexibility components since the six core processes are interconnected and dynamic. Because of this, future research may benefit from comprehensively investigating values with other core processes of psychological flexibility. 
Data Availability Statement The datasets generated during and analyzed during the current study are available from the corresponding author on reasonable request.

\section{Compliance with Ethical Standards}

Informed Consent All procedures followed were in accordance with the ethical standards, and informed consent was obtained from all participants for being included in the study.

\section{References}

Adcock, A. C., Murrell, A. \& Woods, D. (May 2007). Empirical support for the importance of valuing on psychological well-being. Presentation at the annual meeting of the Association for Behavior Analysis.

American Counseling Association (ACA), (2010). Consensus Definition of Counseling. https://www.counseling.org/about-us/about-aca/2020-a-vision-for-the-future-of-counseling/consensus-definition-ofcounseling.

Aydın, Y. (2016). Testing a model of psychological inflexibility, ruminative thinking, worry, and self-compassion in relation to college adjustment. (Unpublished doctoral dissertation). Middle East Technical University, Ankara, Turkey.

Aydın, Y., \& Aydın, G. (2017). Değer Verme Ölçeği (DVÖ)' ni Türk kültürüne uyarlama çalışması. Abant İzet Baysal Üniversitesi Eğitim Fakültesi Dergisi, 17(1), 64-77.

Bond, F. W., Hayes, S. C., Baer, R. A., Carpenter, K. M., Guenole, N., Orcutt, H. K., \& Zettle, R. D. (2011). Preliminary psychometric properties of the acceptance and action questionnaire-II: A revised measure of psychological inflexibility and experiential avoidance. Behavior Therapy, 42(4), 676-688. https://doi.org/10.1016/j.beth. 2011.03.007.

Costa, J., \& Pinto-Gouveia, J. (2013). Experiential avoidance and selfcompassion in chronic pain. Journal of Applied Social Psychology, 43(8), 1578-1591. https://doi.org/10.1111/jasp.12107.

Creswell, J. W. (2013). Qualitative inquiry and research design: Choosing among five approaches ( $3 r d$ ed). Sage Publications.

Dahl, J. C., Plumb, J. C., Stewart, I., \& Lundgren, T. (Eds.). (2009). The art \& science of valuing in psychotherapy: Helping clients discover, explore, and commit to valued action using acceptance and commitment therapy. Inc: Harbinger Publications.

Donald, J. N., Atkins, P. W. B., Parker, P. D., Christie, A., \& Guo, J. (2016). Cognitive defusion predicts more approach and less avoidance coping with stress, independent of threat and self-efficacy appraisals. Journal of Personality, 85(5), 1-46. https://doi.org/10. 1111/jopy.12279.

Forsyth, J. P., Parker, J. D., \& Finlay, C. G. (2003). Anxiety sensitivity, controllability, and experiential avoidance and their relation to drug of choice and addiction severity in a residential sample of substanceabusing veterans. Addictive Behaviors, 28(5), 851-870. https://doi. org/10.1016/S0306-4603(02)00216-2.

Garfield, C. (1992). Second to none: How our smartest companies put people first. Ill. Business One Irwin: Homewood.

Gladding, S. T. (2017). Counseling: A comprehensive profession. Pearson Publications.

Hayes, S. C., \& Lillis, J. (2012). Acceptance and commitment therapy: Theories of psychotherapy series. American Psychological Association.

Hayes, S. C., \& Smith, S. (2005). Get out of your mind into your lifeThe new harbinger.
Hayes, S. C., Strosahl, K. D., Bunting, K., Twohig, M., \& Wilson, K. G. (2004). What is acceptance and commitment therapy? In S. C. Hayes \& K. D. Strosahl (Eds.), A practical guide to acceptance and commitment therapy. Springer.

Hayes, S. C., Strosahl, K., \& Wilson, K. G. (1999). Acceptance and commitment therapy: An experiential approach to behavior change. The Guilford Press.

Hayes, S. C., Strosahl, K. D., \& Wilson, K. G. (2012). Acceptance and commitment therapy: The process and practice of mindful change. Guilford Press.

Hayes, S. C., Wilson, K. G., Gifford, E. V., Follette, V., \& Strosahl, K. (1996). Experiential avoidance and behavioral disorders: A functional dimensional approach to diagnosis and treatment. Journal of Consulting and Clinical Psychology, 64(6), 1152-1168. https://doi. org/10.1037/0022-006X.64.6.1152.

Hernandez, N. C. (2013). Values and valuing in a college population, Unpublished doctoral thesis. TX: University of North Texas.

Kashdan, T. B., Morina, N., \& Priebe, S. (2009). Post-traumatic stress disorder, social anxiety disorder, and depression in survivors of the Kosovo war: Experiential avoidance as a contributor to distress and quality of life. Journal of Anxiety Disorders, 23(2), 185-196. https:// doi.org/10.1016/j.janxdis.2008.06.006.

Kashdan, T. B., \& Rottenberg, J. (2010). Psychological flexibility as a fundamental aspect of health. Clinical Psychology Review, 30(7), 865-878. https://doi.org/10.1016/j.cpr.2010.03.001.

Korkut, F. (2006). Counselor education, program accreditation, and counselor credentialing in Turkey. International Journal for the Advancement of Counseling, 29, 11-20. https://doi.org/10.1007/ s10447-006-9021-6.

Krafft, J., Haeger, J. A., \& Levin, M. E. (2019). Comparing cognitive fusion and cognitive reappraisal as predictors of college student mental health. Cognitive Behavior Therapy, 48(3), 241-252. https://doi.org/10.1080/16506073.2018.1513556.

Krippendorff, K. (2004). Content analysis: An introduction to its methodology (2nd ed.). Sage Publications.

Levin, M. E., Haeger, J., Pierce, B. G., \& Twohig, M. P. (2016). Webbased acceptance and commitment therapy of mental health problems in college students: A randomized controlled trial. Behavior Modification, 41(1), 1-32. https://doi.org/10.1177/ 0145445516659645 .

Levin, M. E., Lillis, J., Seeley, J., Hayes, S. C., Pistorello, J., \& Biglan, A. (2012). Exploring the relationship between experiential avoidance, alcohol use disorders, and alcohol-related problems among first-year college students. Journal of American College Health, 60(6), 443 448. http://doi.org/https://doi.org/10.1080/07448481.2012.673522.

Moustakas, C. (1990). Heuristic research: Design, methodology, and applications. Sage Publications.

Polk, K. L., \& Schoendorff, B. (2014). ACT matrix: A new approach to building psychological flexibility across settings \& populations. New Harbinger.

Reddy, M. K., Pickett, S. M., \& Orcutt, H. K. (2006). Experiential avoidance as a mediator in the relationship between childhood psychological abuse and current mental health symptoms in college students. Journal of Emotional Abuse, 6(1), 67-86. https://doi.org/10. 1300/J135v06n01 04.

Rogers, C. R. (1964). Toward a modern approach to values: The valuing process in the mature person. The Journal of Abnormal and Social Psychology, 68(2), 160-167. https://doi.org/10.1037/h0046419.

Rokeach, M. (1973). The nature of human values. The Free Press.

Schwartz, S. H. (1992). Universals in the content and structure of values: Theoretical advances and empirical tests in 20 countries. In M. P. Zanna (Ed.), Advances in experimental social psychology (Vol. 25, pp. 1-65). Academic Press.

Schwartz, S. H. (2005). Basic human values: Their content and structure across countries. In a. Tamayo \& J. B. Porto (Eds.), Valores e 
comportamento nas organizações [values and behavior in organizations] (pp. 21-55).

Bardi, A., \& Schwartz, S. H. (2003). Values and behavior: Strength and structure of relations. Personality and Social Psychology Bulletin, 29(10), 1207-1220. https://doi.org/10.1177/0146167203254602.

Sheldon, K., Kasser, T., Smith, K., \& Share, T. (2002). Personal goals and psychological growth: Testing an intervention to enhance goal attainment and personality integration. Journal of Personality, 70(1), 5-31. http://doi.org/. https://doi.org/10.1111/1467-6494. 00176 .

Skinner, B. F. (1971). Beyond freedom and dignity. Bantam/Vintage.

Skovholt, T. M. (2012). The counselor's resilient self. Turkish Psychological Counseling and Guidance Journal, 4(38), 137-146.

Skovholt, T., McCarthy, P., \& . (1988). Critical incidents in counselor development [special issue]. Journal of Counseling and Development, 67(2), 69-130.

Smout, M., Davies, M., Burnst, N., \& Christie, A. M. (2014). Development of the valuing questionnaire. Journal of Contextual Behavioral Science, 3(3) http://doi.org/, 164-172. https://doi.org/ 10.1016/j.jcbs.2014.06.001.

Tull, M. T., Gratz, K. L., Salters-Pedneault, K., \& Roemer, L. (2004). The role of experiential avoidance in post-traumatic stress symptoms and symptoms of depression, anxiety, and somatization. Journal of Nervous \& Mental Disease, 192(11), 754-761. https://doi.org/10. 1097/01.nmd.0000144694.30121.89.

Van Dyke, J., Rogers, L., \& Wilson, K. (2006). Valued living, experiential avoidance, and psychological well-being. Paper presented at the presentation at the annual meeting of the Association for Behavior Analysis. GA: Atlanta.

Vilardaga, R., Luoma, J. B., Hayes, S. C., Pistorello, J., Levin, M. E., Hildebrandt, M. J., Kohlenberg, B., Roget, N. A., \& Bond, F. (2011). Burnout among the addiction counseling workforce: The differential roles of mindfulness and values-based processes and work-site factors. Journal of Substance Abuse Treatment, 40(4), 323-335. https://doi.org/10.1016/j.jsat.2010.11.015.
Wilson, K. G., \& Murrell, A. R. (2004). Values work in acceptance and commitment therapy: Setting a course for behavioral treatment. In S. C. Hayes, V. M. Follette, \& M. M. Linehan (Eds.), Mindfulness and acceptance: Expanding the cognitive-behavioral tradition, 120 151. Guilford Press.

Wilson, K. G., \& Dufrene, T. (Collaborator). (, 2008). Mindfulness for two: An acceptance and commitment therapy approach to mindfulness in psychotherapy. New Harbinger Publications.

Wilson, K. G., Sandoz, E. K., Kitchens, J., \& Roberts, M. E. (2010). The valued living questionnaire: Defining and measuring valued action within a behavioral framework. The Psychological Record, 60, 249272. https://doi.org/10.1007/BF03395706.

Wilson, K. G., Sandoz, E. K., Kitchens, J., \& Roberts, M. (2010). The valued living questionnaire: Defining and measuring valued action within a behavioral framework. The Psychological Record, 60(2), 249-272. https://doi.org/10.1007/BF03395706.

Yavuz, F., Ulusoy, S., Iskın, M., Esen, F. B., Burhan, H. S., Karadere, M. E., \& Yavuz, N. (2016). Turkish version of acceptance and action questionnaire-II (AAQ-II): A reliability and validity analysis in clinical and non-clinical samples. Bulletin of Clinical Psychopharmacology, 26(4), 397-408. https://doi.org/10.5455/ bcp. 20160223124107.

Yin, R. K. (2003). Case study research: Design and methods (3rd ed.) Thousand Oaks.

Zettle, R., Hocker, T. R., Mick, K., Scofield, B. E., Petersen, C. L., Song, H., \& Sudarijanto, R. P. (2005). Differential strategies in coping with pain as a function of level of experiential avoidance. The Psychological Record, 55(4) http://doi.org/, 511-524. https://doi. org/10.1007/BF03395524.

Publisher's Note Springer Nature remains neutral with regard to jurisdictional claims in published maps and institutional affiliations. 